\title{
Isolation and screening of methicillin-resistant Staphylococcus aureus from health care workers in Libyan hospitals
}

\author{
M.O. Ahmed, ${ }^{1,2}$ A.K. Elramalli, ${ }^{3}$ S.G. Amri, ${ }^{4}$ A.R. Abuzwedd ${ }^{5}$ and Y.M. Abouzeed ${ }^{1,2}$
}

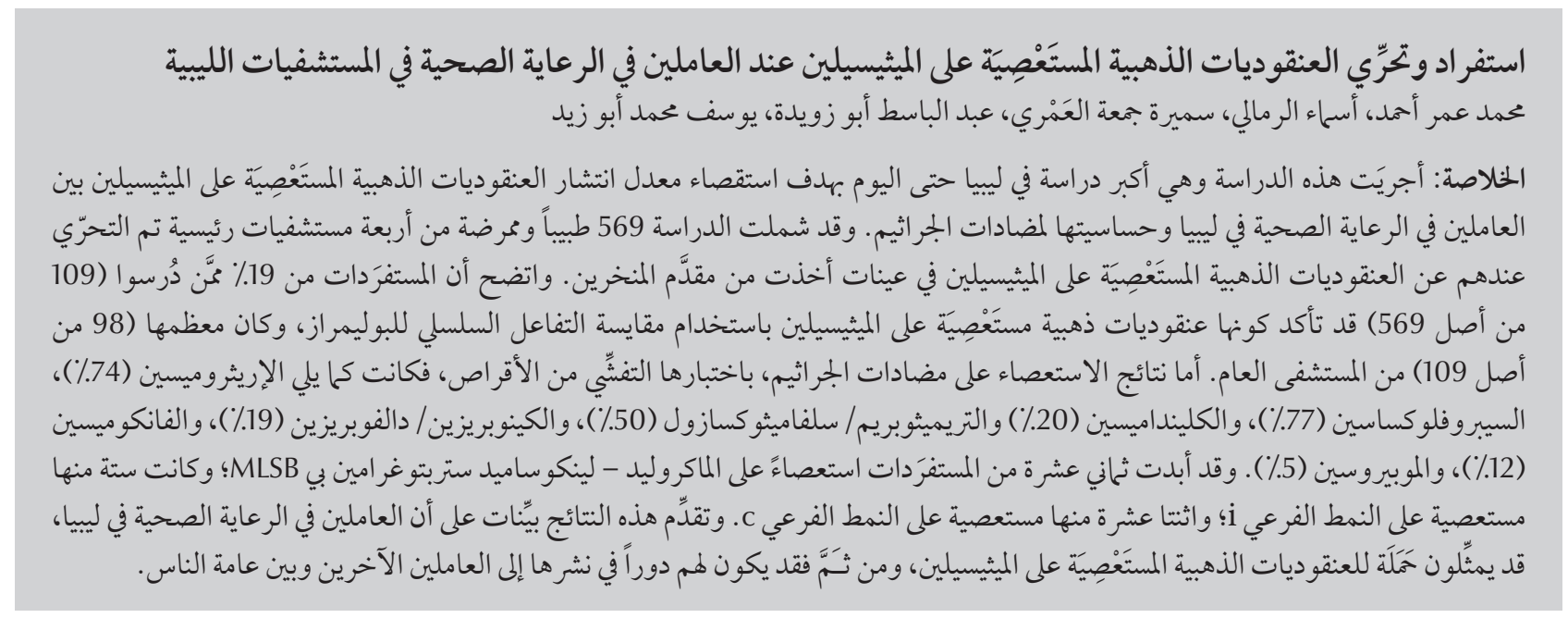

ABSTRACT This is the largest Libyan study to date to investigate the prevalence and antimicrobial susceptibility of methicillin-resistant Staphylococcus aureus (MRSA) among health care workers in Tripoli, Libya. A total of 569 doctors and nurses from 4 main hospitals were screened for MRSA with specimens collected from the anterior nares. Isolates from 109 of the 569 subjects (19\%) were confirmed as MRSA by polymerase chain reaction assay; the majority (98/109) were from a general hospital. Antimicrobial resistance patterns tested by disk diffusion were as follows: erythromycin (74\%), ciprofloxacin (77\%), clindamycin (20\%), trimethoprim/sulfamethoxazole (50\%), quinuprisin/dalfopristin (19\%), vancomycin (12\%) and mupirocin (5\%). Eighteen isolates exhibited macrolide-lincosamide-streptogramin B resistance (MLSB): 6 were MLSBi and 12 were MLSBc. The results provide evidence that Libyan health care workers could serve as MRSA carriers and play a role in the dissemination of MRSA to the public and other workers.

Isolement et dépistage de Staphylococcus aureus résistant à la méticilline chez des agents de santé dans des hôpitaux libyens

RÉSUMÉ II s'agit de l'étude libyenne la plus importante à ce jour, évaluant la prévalence et la sensibilité aux antimicrobiens de Staphylococcus aureus résistant à la méticilline chez des agents de santé à Tripoli (Libye). Le dépistage a été réalisé auprès de 569 médecins et membres du personnel infirmier travaillant dans quatre grands hôpitaux, au moyen d'un prélèvement à partir des fosses nasales. Des isolats de 109 sujets sur 569 (19 \%) ont été confirmés comme étant des souches de Staphylococcus aureus résistant à la méticilline, au moyen de l'amplification en chaîne par polymérase ; la majorité (98 sur 109) provenait d’un hôpital général. Les profils de résistance aux antimicrobiens analysés par la méthode de diffusion en disques étaient les suivants : érythromycine (74\%), ciprofloxacine $(77 \%)$, clindamycine (20\%), triméthoprime/sulfaméthoxazole (50\%), dalfopristine/quinupristine (19\%), vancomycine (12\%) et mupirocine (5\%). Dix-huit isolats présentaient une résistance aux streptogramines de type B, aux macrolides et aux lincosamides : six avaient une résistance inductible aux streptogramines de type B, aux macrolides et aux lincosamides et douze avaient une résistance constitutive. Les résultats prouvent que les agents de santé libyens peuvent servir de porteurs de Staphylococcus aureus résistant à la méticilline et jouer un rôle dans sa diffusion à la population et aux autres agents. 


\section{Introduction}

Methicillin-resistant Staphylococcus aureus (MRSA) first emerged as a nosocomial pathogen in the early 1960s and it continues to be a significant public health concern $[1,2]$. Resistance to methicillin in staphylococci is mediated by an altered penicillin-binding protein (PBP2a), which is encoded by the mecA gene and confers resistance to most of the current $\beta$-lactam antimicrobial agents [2]. The genetic plasticity of $S$. aureus has resulted in the emergence of methicillin resistance in different strains with varying degrees of antibiotic resistance and virulence patterns $[3,4]$.

There are major international concerns about rising levels of MRSA and multi-drug resistant $S$. aureus owing to the difficulties of treating infections and the ease with which MRSA spreads in hospitals [5]. Until recently, most MRSA infections were acquired primarily in hospital settings. Now MRSA is responsible for both hospital and community-acquired infections [1]. Since the first reports of community-associated MRSA infections emerged in the early 1980s, studies have shown an increase in the circulation of MRSA infections beyond hospital settings [6].

The role of health care workers (HCWs) in the nosocomial transmission of MRSA has been widely discussed, with many studies citing HCWs as a source of nosocomial transmission of MRSA in developing countries $[7,8]$ including Libya [9-14]. Pre-intervention studies of HCW colonization play an important role in providing evidence about the spread of MRSA [15].

In the current study we investigated the prevalence and antimicrobial susceptibility profiles of MRSA among HCWs in the main hospitals in Tripoli, Libya.

\section{Methods}

\section{Sources of samples}

Four major different hospitals were included in this study and were assigned the following abbreviations: A, B, C, and D. Hospital A was a general community-based hospital that deals with a variety of different health conditions; hospitals B, C and D were acute care paediatric, emergency and eye surgery hospitals respectively.

\section{Samples and data collection}

Samples were collected from 569 HCWs (215 doctors and 354 nurses) present in the 4 hospitals from January-July 2009. All HCWs present at the time of sampling and consenting to participate were included. Samples were taken from the anterior nares of consenting HCWs as follows: a single culturette swab was moisturized in sterile water and then gently rotated inside both nares. Specimens were immediately sent to the laboratory and processed within 1 hour of sampling. Contact details were collected from the HCWs for follow-up of any possible positive MRSA but these were kept confidential from the hospital. Positive MRSA carriers were approached confidentially for follow-up.

\section{Isolation, identification and confirmation of MRSA}

MRSA was isolated by direct plating of swabs onto mannitol salt agar (MSA) containing $2 \mathrm{mg} / \mathrm{L}$ oxacillin (Oxoid), followed by incubation for $48 \mathrm{~h}$ at 35 ${ }^{\circ} \mathrm{C}$. Suspected individual colonies were selected from each MSA plate and subcultured and grown on Columbia agar supplemented with 5\% horse blood (Oxoid), incubated as above and examined after $24 \mathrm{~h}$. Suspected isolates were preliminarily subjected to Gram stain and test strips (API Staph, bioMérieux) for identification of presumptive S. aureus. Isolates were then tested by disk diffusion susceptibility testing, in accordance with the British Society for Antimicrobial Chemotherapy guidelines (BSAC), using cefoxitin, as described by Andrews [16], and the presence of PBP2a was detected by latex agglutination testing for PBP2a (Oxoid). Isolates that scored positive with both BSAC-disk diffusion test and the latex agglutination test for PBP2a were screened by PCR $[17,18]$ to amplify the femA and $m e c A$ genes to definitively confirm MRSA based on species and resistance level, respectively. Modified protocols for both PCR reactions were based on a personal communication with $\mathrm{N}$. Williams from the University of Liverpool. Confirmed MRSA isolates, based on previous testing and PCR confirmation, were further analysed and subjected to antimicrobial susceptibility testing based on updated testing information obtained from the BSAC website.

\section{Susceptibility testing of MRSA}

Both the disk diffusion susceptibility test and agar dilution method were applied according to BSAC recommendations. The MRSA isolates were tested by the disk diffusion susceptibility method [16] against: oxacillin, trimethoprim/ sulfamethoxazole, erythromycin, clindamycin, ciprofloxacin, mupirocin, quinupristin/dalfopristin, vancomycin and linezolid. Minimum inhibitory concentrations (MICs) were determined by the agar dilution method [19] only for isolates that exhibited resistance to vancomycin and mupirocin in the disk diffusion susceptibility tests. Disk diffusion tests were performed on isolates exhibiting erythromycin resistance to assay for inducible clindamycin resistance [macrolide-lincosamide-streptogramin B inducible resistance (MLSBi) phenotype], as described by Fiebelkorn et al. [20]. In-house confirmed MRSA strains, which were previously genotyped by PCR $[17,18]$, served as positive controls throughout the study. All MRSA samples and isolates were stored at $-80^{\circ} \mathrm{C}$. 


\section{Results}

\section{Prevalence of MRSA among HCWs}

A total of 569 HCWs (215 doctors and 354 nurses) from 4 different hospitals in Tripoli were tested for MRSA. Out of the 569 HCWs, 128 (22\%) were characterized as positive for MRSA by laboratory methods and 109 (19\%) were confirmed as MRSA by PCR (Table 1). Three hospitals scored positive for confirmed MRSA with some differences between hospitals; $21 \%$ (98/473) of isolates from HCWs at the general hospital were positive, $9 \%$ $(3 / 32)$ at the paediatric hospital, $21 \%$ $(8 / 39)$ at the eye surgery hospital and $0 \%(0 / 25)$ at the emergency hospital.

\section{Susceptibility testing for MRSA}

All confirmed isolates exhibited resistance to oxacillin. Out of the 109 confirmed MRSA isolates, 81 (74\%) were resistant to erythromycin, and disk diffusion tests showed that 6 of those isolates (6\%) exhibited inducible resistance to clindamycin (MLSBi phenotype). Only 18 of the 33 clindamycin-resistant strains expressed an MLSB phenotype according to BSAC guidelines. Twelve isolates (11\%) were characterized as having an MLSBc (constitutive) phenotype. Only 5 (5\%) isolates showed resistance to mupirocin by the disk diffusion susceptibility test, and MIC analysis confirmed that only 1 isolate was highly resistant to mupirocin (MIC $\geq 512 \mathrm{mg} / \mathrm{mL}$ ). Overall, 84 isolates (77\%) were identified as ciprofloxacin resistant; 82 of these were from isolates taken from HCWs at the general hospital. Resistance to trimethoprim/ sulfamethoxazole was exhibited by 55 isolates (50\%). MIC analysis showed that none of the 13 isolates initially identified as vancomycin resistant by the disk diffusion susceptibility test were vancomycin resistant. None of the confirmed MRSA isolates showed resistance to linezolid. The majority of the resistant phenotypes were identified among general hospital isolates (Table 2).

\section{Discussion}

Overall the proportion of confirmed MRSA-positive samples was 19\% (109/569), with some differences between hospitals: $21 \%$ of HCWs from the general hospital (hospital A), and between $0 \%$ and $21 \%$ of HCWs from the specialist hospitals (hospital B, hospital C and hospital D). High levels of MRSA were found among HCW samples from the general hospital (hospital A). This hospital is the main and biggest hospital in Tripoli and Libya and therefore had a much larger sample size than the other 3 hospitals. Hospitals B, C and D, in which significantly fewer HCWs were tested $(32,25$ and 39 respectively), showed more variable levels of colonization $(9 \%, 0 \%$ and $21 \%$ respectively). The specialist hospitals deal with specific clinical conditions and therefore the overall numbers of HCWs, patients and visitors were less than those in the much larger general hospital. Both hospital size and other factors, such as antibiotic therapy, and hospital management are likely to be of importance in the dissemination and development of MRSA infections.

Previous studies have shown the role of clinical staff as nasal carriers of MRSA $[9,21]$. Nares and the anterior nare are the most important sites of staphylococcal colonization and potential sources of MRSA [9,22]. Screening for nasal carriers and colonized patients is very effective in controlling the spread of MRSA [22]. MRSA rates in the current study are likely to be underestimated, since we only used nasal swabs and did not test other sites, such as the throat. Previously in Libya the prevalence of MRSA was reported to be around 39\% [9] and misidentification of MRSA in Libyan hospitals have been also reported [10]; in our study a lower prevalence was reported using genotyping PCR confirmation. The importance of genotyping methods in determining the possible transmission of MRSA from HCWs to patients has also been demonstrated [7,23]. Unknown carriers among patients may introduce MRSA into the hospital and serve as a source of transmission to other patients, primarily via the transiently colonized hands of hospital staff. An investigation by Vonberg et al. indicated that screening of HCWs should be performed before starting work duties in order to prevent the detection of transient, shortterm MRSA carriage that may occur during a work shift [24]. However, such

\begin{tabular}{|c|c|c|c|c|c|}
\hline \multirow[t]{2}{*}{ Source } & \multirow{2}{*}{$\begin{array}{c}\text { Total tested } \\
\text { No. }\end{array}$} & \multicolumn{2}{|c|}{ Positive for MRSA } & \multicolumn{2}{|c|}{ PCR-confirmed MRSA } \\
\hline & & No. & $\%$ & No. & $\%$ \\
\hline Hospital A & 473 & 117 & 25 & 98 & 21 \\
\hline Hospital B & 32 & 3 & 9 & 3 & 9 \\
\hline Hospital C & 25 & 0 & 0 & 0 & 0 \\
\hline Hospital D & 39 & 8 & 21 & 8 & 21 \\
\hline Total & 569 & 128 & 22 & 109 & 19 \\
\hline
\end{tabular}

Hospital $A$ = general hospital; hospital $B=$ paediatric hospital; hospital $C=$ emergency hospital; hospital $D=$ eye surgery hospital. 


\begin{tabular}{|c|c|c|c|c|c|c|}
\hline \multirow[t]{3}{*}{ Antibiotic } & \multicolumn{6}{|c|}{ Source } \\
\hline & \multirow{2}{*}{$\begin{array}{c}\text { Hospital A } \\
\text { No. }\end{array}$} & \multirow{2}{*}{$\begin{array}{c}\text { Hospital B } \\
\text { No. }\end{array}$} & \multirow{2}{*}{$\begin{array}{c}\text { Hospital C } \\
\text { No. }\end{array}$} & \multirow{2}{*}{$\begin{array}{c}\text { Hospital D } \\
\text { No. }\end{array}$} & \multicolumn{2}{|c|}{ All hospitals } \\
\hline & & & & & No. & $\%$ \\
\hline Oxacillin & 98 & 3 & 0 & 8 & 109 & 100 \\
\hline Ciprofloxacin & 82 & 1 & 0 & 1 & 84 & 77 \\
\hline Erythromycin & 77 & 2 & 0 & 2 & 81 & 74 \\
\hline Trimethoprim/sulfamethoxazole & 53 & 1 & 0 & 1 & 55 & 50 \\
\hline Clindamycin & 31 & 1 & 0 & 1 & 33 & 30 \\
\hline Quinupristin/dalfopristin & 21 & 0 & 0 & 0 & 21 & 19 \\
\hline Vancomycin & 12 & 1 & 0 & 0 & 13 & 12 \\
\hline Mupirocin & 5 & 0 & 0 & 0 & 5 & 5 \\
\hline $\mathrm{MLSB}_{i}$ & 6 & 0 & 0 & 0 & 6 & 6 \\
\hline $\mathrm{MLSB}_{c}$ & 11 & 1 & 0 & 0 & 12 & 11 \\
\hline Total & 98 & 3 & 0 & 8 & 109 & 100 \\
\hline
\end{tabular}

Hospital $A=$ general hospital; hospital $B=$ paediatric hospital; hospital $C=$ emergency hospital; hospital $D=$ eye surgery hospital . $M L S B_{i}=$ macrolide-lincosamide-streptogramin $B$ inducible resistance; $M L S B_{c}$ macrolide-lincosamide-streptogramin $B$ constitutive resistance.

data on MRSA in Libya are not currently available and this level of screening was beyond the scope of the current study. MRSA transmission between hospitals has also been documented [25], and healthy individuals with nasal MRSA colonization are more likely to develop an infection after having medical procedures performed in the hospital $[7,26]$. Further genotypic analysis of HCW isolates and comparisons with clinical samples would yield important information regarding MRSA transmission; such information on clinical MRSA isolates is not currently available in Libyan hospitals.

Risk factors associated with MRSA carriage among HCWs, such as chronic skin diseases and poor hygiene, have been reported [7]. Similarly, patients with surgical wounds, urinary tract infections and skin infections were at high risk for MRSA carriage and infection [27]. Cross- and autoinfection by the hands of HCW carriers, appear to be the most common routes for MRSA infection [27]. The eradication of MRSA carriage and colonization among HCWs is possible [7], and our results suggest that the hospitals included in this study should implement protocols to control the risk of nosocomial MRSA, such as routine screening of HCWs for MRSA, susceptibility testing of isolates obtained, and education, hand disinfection and temporary layoff for staff [24].

Agar screening methods and latex agglutination of PBP2a are reported to be highly specific in identifying MRSA [16] and they are a practical alternative when PCR facilities are not readily available for detecting MRSA. In this study these methods proved to be efficient. However, PCR is still the golden standard tool for detecting and confirming MRSA. The disk diffusion susceptibility method is a reliable test used to guide therapy, monitor resistance trends and evaluate MRSA susceptibility [10,28] and is valuable for detecting inducible clindamycin resistance in erythromycin resistant strains $[11,18,29]$. However, disk diffusion susceptibility testing is not reliable for detecting vancomycinresistant staphylococci, and MIC determination is recommended for determining glycopeptide resistance. In the current study, we did not identify any confirmed vancomycin resistance by MIC analysis; future experiments will include the application of other accurate tests such as dry chemistry technology $\left(\right.$ Etest $\left.^{\circ}\right)$ [16]. Although resistance to the important antimicrobial drug vancomycin has not been widely reported, limitations in its use for treating S. aureus infection are emerging [30]. However, drugs such as daptomycin and linezolid remain valuable [31].

In the current study, a high proportion of the confirmed MRSA isolates (77\%) exhibited resistance to flouroquinolones. Resistance to mupirocin was only present in a small proportion of confirmed MRSA isolates, and only 1 of the isolates exhibited a high level of mupirocin resistance (MIC $\geq 512$ $\mathrm{mg} / \mathrm{mL}$ ). Mupirocin ointment is the drug of choice for treating nasal MRSA colonization and it has been reported to be effective in eliminating MRSA colonization [7]. However, new MRSA colonization has been reported even after the use of mupirocin [26].

Macrolide and lincosamide resistance are increasingly reported among MRSA isolates. Previously MRSA-MLSB phenotypes have been reported in Libya $[11,14]$. MRSA can severely compromise therapy and is associated with failed clindamycin treatment of MRSA infections [31]. The large proportion of isolates that were clindamycin susceptible in the current study (70\%) suggests that the MRSA isolates from $\mathrm{HCW}$ s were most 
likely community-acquired strains. Just over half of the clindamycin-resistant strains expressed an MLSB phenotype. The current data and others indicate that clindamycin could still be used to treat MRSA infections in our hospitals, although susceptibility testing for the detection of inducible resistance to clindamycin should be routinely performed [11]. Future studies should focus on determining the rate of MRSA transmission between HCWs and patients.
To our knowledge, the current study is the largest to date to analyse the prevalence and antimicrobial susceptibility of MRSA among HCWs in Libyan hospitals. The results of the current study highlight the need for infection control programmes and antibiotic monitoring in Libyan hospitals; it also reveals an urgent need for additional MRSA-focused studies in Libya. The data regarding antimicrobial resistance profiles of MRSA presented here provide important information for clinicians and epidemiologist and stress the need for a national MRSA monitoring system.

\section{Acknowledgements}

The authors would like to thank all of the staff at the participating hospitals who assisted and contributed to this study. We thank the National Authority for Scientific Research, Tripoli, Libya, for their financial and unlimited support to continue our research in this filed.

\section{References}

1. Robinson JO et al. Community-associated versus healthcareassociated methicillin-resistant Staphylococcus aureus bacteraemia: a 10-year retrospective review. European Journal of Clinical Microbiology \& Infectious Diseases, 2009, 28:353-361.

2. Palavecino E. Clinical, epidemiological, and laboratory aspects of methicillin-resistant Staphylococcus aureus (MRSA) infections. Methods in Molecular Biology, 2007, 391:1-19.

3. Holden MT et al. Complete genomes of two clinical Staphylococcus aureus strains: Evidence for the rapid evolution of virulence and drug resistance. Proceedings of the National Academy of Sciences of the United States of America, 2004, 101:9786-9791.

4. de Lencastre $\mathrm{H}$ et al. Antibiotic resistant Staphylococcus aureus: a paradigm of adaptive power. Current Opinion in Microbiology, 2007, 10:428-435.

5. Gould IM. The clinical significance of methicillin-resistant Staphylococcus aureus. Journal of Hospital Infection, 2005, 61:277-282.

6. Groom AV et al. Community-acquired methicillin-resistant Staphylococcus aureus in a rural american indian community. Journal of the American Medical Association, 2001, 286:12011205.

7. Albrich WC, Harbarth S. Health-care workers: source, vector, or victim of MRSA? Lancet Infectious Diseases, 2008, 8:289-301.

8. Goyal $\mathrm{R}$ et al. Colonisation of methicillin resistant $S$. aureus among health care workers in a tertiary care hospital of Delhi. Indian Journal of Medical Sciences, 2002, 56:321-324.

9. Zorgani A et al. Detection of meticillin-resistant Staphylococcus aureus among healthcare workers in Libyan hospitals. Journal of Hospital Infection, 2009, 73:91-92.

10. Ahmed MO et al. Misidentification of methicillin-resistant Staphylococcus aureus (MRSA) in hospitals in Tripoli, Libya. Libyan Journal of Medicine, 2010, 5:5230.

11. Ahmed MO et al. Detection of inducible clindamycin resistance (MLSB(i)) among methicillin-resistant Staphylococcus aureus (MRSA) from Libya. Libyan Journal of Medicine, 2010, 5:4636.

12. Belgasim Z, Saadaoui A, Zorgani A. Screening for methicillinresistant Staphylococcus aureus among health care workers in the African Oncology Institute, Sabrata-Libya. American Journal of Infection Control, 2010, 38:498-499.

13. Ghenghesh KS et al. Isolation of methicillin-resistant Staphylococcus aureus (MRSA) from rented DVDs. American Journal of Infection Control, 2009, 37:612.
14. Zorgani A et al. Inducible clindamycin resistance among staphylococci isolated from burn patients. Libyan Journal of Medicine, 2009, 4:104-106.

15. Amorim ML et al. Epidemiology of methicillin-resistant Staphylococcus aureus (MRSA) nasal colonization among patients and healthcare workers in a portuguese hospital: A pre-intervention study toward the control of MRSA. Microbial Drug Resistance (Larchmont, N.Y.), 2009, 15:19-26.

16. Andrews JM. BSAC standardized disc susceptibility testing method (version 7). Journal of Antimicrobial Chemotherapy, 2008, 62:256-278.

17. Francois P et al. Rapid detection of methicillin-resistant Staphylococcus aureus directly from sterile or non-sterile clinical samples by a new molecular assay. Journal of Clinical Microbiology, 2003, 41:254-260.

18. Vannuffel $\mathrm{P}$ et al. Specific detection of methicillin-resistant Staphylococcus species by multiplex PCR. Journal of Clinical Microbiology, 1995, 33:2864-2867.

19. Andrews JM. Determination of minimum inhibitory concentrations. Journal of Antimicrobial Chemotherapy, 2001, 48 (Suppl. 1):5-16.

20. Fiebelkorn KR et al. Practical disk diffusion method for detection of inducible clindamycin resistance in Staphylococcus aureus and coagulase-negative staphylococci. Journal of Clinical Microbiology, 2003, 41:4740-4744.

21. Johnston CP et al. Staphylococcus aureus colonization among healthcare workers at a tertiary care hospital. Infection Control and Hospital Epidemiology, 2007, 28:1404-1407.

22. Mathanraj S et al. Screening for methicillin-resistant Staphylococcus aureus carriers among patients and health care workers of a tertiary care hospital in south India. Indian Journal of Medical Microbiology, 2009, 27:62-64.

23. Dimitrov T et al. Point surveillance of Staphylococcus aureus carriage among medical staff in infectious diseases hospital, Kuwait. Medical Principles and Practice, 2003, 12:139-144.

24. Vonberg RP et al. How often do asymptomatic healthcare workers cause methicillin-resistant Staphylococcus aureus outbreaks? A systematic evaluation. Infection Control and Hospital Epidemiology, 2006, 27:1123-1127.

25. Ayliffe GA. The progressive intercontinental spread of methicillin-resistant staphylococcus aureus. Clinical Infectious Diseases, 1997, 24(Suppl 1):S74-S79.

26. Ellis MW et al. Targeted intranasal mupirocin to prevent colonization and infection by community-associated methicillin- 
resistant Staphylococcus aureus strains in soldiers: a cluster randomized controlled trial. Antimicrobial Agents and Chemotherapy, 2007, 51:3591-3598.

27. Coello $R$ et al. Prospective study of infection, colonization and carriage of methicillin-resistant Staphylococcus aureus in an outbreak affecting 990 patients. European Journal of Clinical Microbiology \& Infectious Diseases, 1994, 13:74-81.

28. Potz NA et al. Reliability of routine disc susceptibility testing by the British Society for Antimicrobial Chemotherapy (BSAC) method. Journal of Antimicrobial Chemotherapy, 2004, 53:729-738.

29. Oğuz VA et al. Tafilokokoklarda induklenebilir klindamisin direnci ve diger antibiyotiklere duyarlilik oranlari. [The rate of inducible clindamycin resistance and susceptibilities to other antimicrobial agents in staphylococci]. Mikrobiyoloji Bulteni, 2009, 43:37-44.

30. Tenover FC et al. Increasing resistance to vancomycin and other glycopeptides in Staphylococcus aureus. Emerging Infectious Diseases, 2001, 7:327-332.

31. Lewis JS, Ellis MW. Approaches to serious methicillin-resistant Staphylococcus aureus infections with decreased susceptibility to vancomycin: clinical significance and options for management. Current Opinion in Infectious Diseases, 2007, 20:568-573.

\section{Antimicrobial resistance}

Many infections can no longer be treated effectively with common anti-infective drugs. Resistance poses a growing threat to the treatment and control of infectious diseases, ranging from those that have long been endemic in human populations - malaria, tuberculosis, and sexually transmitted infections - to more recent pandemics such as the one due to human immunodeficiency virus (HIV) or the influenza virus (A H1N1 2009). Antimicrobial resistance threatens the control of several other community-acquired infections. An alarming proportion of health care-associated infections are also caused by resistant pathogens.

To tackle this problem, the World Health Organization (WHO) has made antimicrobial resistance an organizationwide priority and made it the focus of World Health Day 2011. In addition, WHO will publish in the near future a reference book entitled Combating antimicrobial resistance: experiences from the field describing the need for and examples of successful interventions.

Further information about the work of WHO in combating the growing problem of antimicrobial resistance can be found at: http://www.who.int/topics/drug_resistance/en/ 